University of Nebraska - Lincoln

DigitalCommons@University of Nebraska - Lincoln

Mechanical \& Materials Engineering Faculty

Publications

Mechanical \& Materials Engineering,

Department of

4-26-2011

\title{
Transverse permeability of fibrous porous media
}

\author{
Ali Tamayol \\ University of Nebraska-Lincoln \& Simon Fraser University, atamayol@uchc.edu \\ Majid Bahrami \\ Simon Fraser University, mbahrami@sfu.ca
}

Follow this and additional works at: https://digitalcommons.unl.edu/mechengfacpub

Part of the Mechanics of Materials Commons, Nanoscience and Nanotechnology Commons, Other Engineering Science and Materials Commons, and the Other Mechanical Engineering Commons

Tamayol, Ali and Bahrami, Majid, "Transverse permeability of fibrous porous media" (2011). Mechanical \& Materials Engineering Faculty Publications. 313.

https://digitalcommons.unl.edu/mechengfacpub/313

This Article is brought to you for free and open access by the Mechanical \& Materials Engineering, Department of at DigitalCommons@University of Nebraska - Lincoln. It has been accepted for inclusion in Mechanical \& Materials Engineering Faculty Publications by an authorized administrator of DigitalCommons@University of Nebraska Lincoln. 


\title{
Transverse permeability of fibrous porous media
}

\author{
Ali Tamayol* and Majid Bahrami ${ }^{\dagger}$ \\ Mechatronic Systems Engineering, School of Engineering Science, Simon Fraser University, Surrey, BC, Canada
}

(Received 6 August 2010; revised manuscript received 29 November 2010; published 26 April 2011)

\begin{abstract}
In this study, the transverse permeability of fibrous porous media is studied both experimentally and theoretically. A scale analysis technique is employed for determining the transverse permeability of various fibrous matrices including square, staggered, and hexagonal arrangements of unidirectionally aligned fibers, as well as simple two-directional mats and simple cubic structures. In the present approach, the permeability is related to the porosity, fiber diameter, and tortuosity of the medium. In addition, the pressure drop in several samples of tube banks of different arrangements and metal foams are measured in the creeping flow regime. The pressure-drop results are then used to calculate the permeability of the samples. The developed compact relationships are successfully verified through comparison with these experimental results and the data reported by others. Our results suggest that fiber orientation has an important effect on the permeability; however, these effects are more pronounced in low porosities, i.e., $\varepsilon<0.7$.
\end{abstract}

DOI: 10.1103/PhysRevE.83.046314

PACS number(s): 47.56.+r, 47.15.G-

\section{INTRODUCTION}

Study of flow in fibrous porous media is important in many natural and industrial processes such as physiological transport phenomena [1], filtration [2-4], composite fabrication [5,6], compact heat exchangers [7,8], paper production [9], and fuel cell technology $[10,11]$. As such, prediction of the flow properties of fibrous materials, including permeability and inertial coefficient, has drawn the attention of numerous researchers. Authors have employed various theoretical and experimental techniques to investigate the problem. Comprehensive reviews of the pertinent literature can be found in Refs. $[4,6,12]$. Permeability, which can be interpreted as the flow conductance of the solid matrix, is related to geometrical features of the solid matrix, including particle size and shape, pore size, and pore distribution.

Fibrous materials can be divided into one-, two-, and three-directional media (see Fig. 1). In one-directional (1D) structures, the axes of fibers are parallel to each other. In twodirectional (2D) fibrous matrices, the axes of fibers are located on planes parallel to each other with random positions and orientations on these planes. The axes of fibers in three-directional (3D) structures are randomly positioned and oriented in space. $1 \mathrm{D}$ and 2D materials are anisotropic, e.g., see [13,14] for more details. However, 3D structures can be considered isotropic, e.g., metal-foam samples studied in this study. The considered microstructures are indeed anisotropic; thus, this study can only be applied to transverse flows. Transverse direction for $1 \mathrm{D}$ fibers is normal to the fibers' axes, and for 2D materials is perpendicular to the fibers' planes [12]. In isotropic 3D structures, transverse flow is perpendicular to any plane.

Due to the complex geometry and their random nature, developing exact solutions for flow and permeability of actual fibrous materials is highly unlikely. Nonetheless, to perform preliminary design and optimization and, more importantly, to recognize and investigate the key parameters involved in the flow properties, approximate solutions often suffice.

\footnotetext{
*ali_tamayol@sfu.ca

†mbahrami@sfu.ca
}

To estimate the permeability of the fibrous structures, several researchers have modeled the complex microstructure of the porous media with simplified 1D "unit cells" [15-23]. The models developed theoretically for transverse permeability of 1D structures are plotted against experimental data, collected from various sources, in Fig. 2. Most of the listed models have limited range of application as reported by others. It can be seen from Fig. 2 that the model of Tamayol and Bahrami [21] captures the trends of experimental data over the entire range of porosity. However, the model of Tamayol and Bahrami [21] is limited to square arrays of fibers.

Theoretical studies of permeability of 2D and 3D materials are not as frequent as 1D arrangements, which is, in part, due to the geometrical complexity of these media. A selection of the existing models for $2 \mathrm{D}$ and $3 \mathrm{D}$ structures [2,12,2426] are plotted and compared with existing experimental data collected from several sources in Figs. 3 and 4, respectively. It can be seen that the existing models are not accurate and can not be used over the entire range of porosity.

Therefore, the objectives of this paper are as follows:

(i) Develop a theoretical approach that is applicable to $1 \mathrm{D}$, $2 \mathrm{D}$, and $3 \mathrm{D}$ fibrous matrices and can accurately capture the trends observed in experimental data.

(ii) Investigate the effects of relevant geometrical parameters involved and identify the controlling parameters.

(iii) Perform an independent experimental study on permeability of fibrous structures to verify the developed models.

A scale analysis technique is employed to predict the permeability of a variety of unit cells including square, staggered, and hexagonal arrangements of 1D fibers, simple 2D mats, and simple cubic structures. This method, which was originally applied to fibrous media by Clague et al. [27], is modified to improve its accuracy. Moreover, compact relationships are presented for determining the permeability of each category of fibrous porous media, i.e., 1D, 2D, and $3 \mathrm{D}$, as a function of porosity and fiber diameter. In addition, pressure drop is measured for creeping flow through several samples of tube banks and aluminum foams with 1D and 3D structures, respectively. The developed solutions are successfully compared with experimental and numerical results for a wide range of geometries and materials. 
(a)

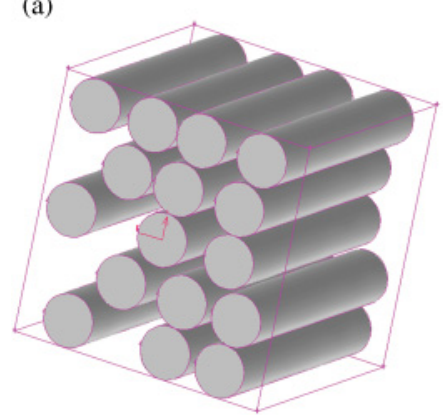

(b)

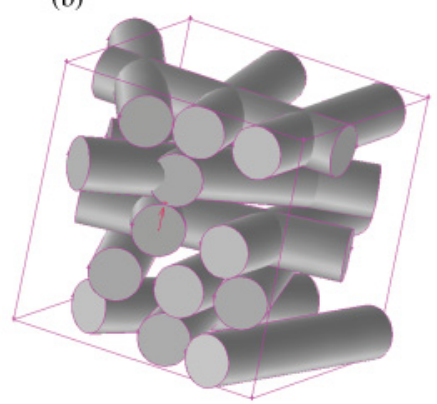

(c)

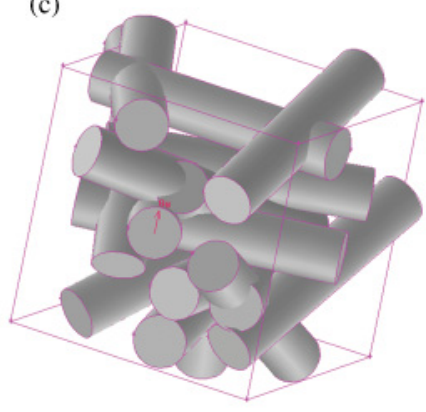

FIG. 1. (Color online) Structures with different fibers orientation; (a) 1 directional (1D), (b) 2 directional (2D), and (c) 3 directional (3D).

\section{GEOMETRICAL MODELING}

Based on the orientation of fibers in the medium, the fibrous matrix can be divided into $1 \mathrm{D}, 2 \mathrm{D}$, or $3 \mathrm{D}$ structures. The simplest representation of $1 \mathrm{D}$ structures or generally fibrous media is the ordered arrangements of unidirectionally aligned cylinders. In this paper, several ordered structures including square, staggered, and hexagonal arrays of fibers are considered where flow is normal to fibers axes (see Fig. 5). The solid volume fractions $\varphi$ for the arrangements shown in Fig. 5 are related to the distance between the centers of adjacent fibers $S$ and the fibers' diameter $d$ :

$$
\varphi= \begin{cases}\frac{\pi d^{2}}{4 S^{2}} & \text { Square } \\ \frac{\pi d^{2}}{2 \sqrt{3} S^{2}} & \text { Staggered } \\ \frac{\pi d^{2}}{3 \sqrt{3} S^{2}} & \text { Hexagonal }\end{cases}
$$

To study woven or textile structures with nonoverlapping fibers, the geometry shown in Fig. 6 is considered where fluid

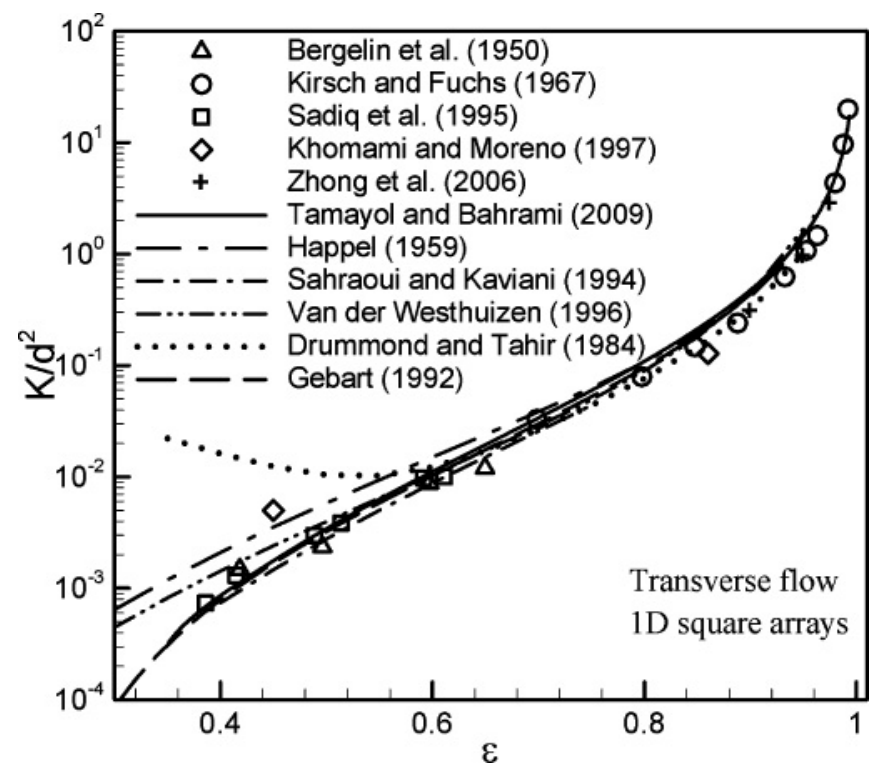

FIG. 2. Comparison of the existing models for square arrangements with experimental data collected from various sources. flow is normal to the fibers planes. The following relationship exists between $\varphi$ and other geometrical parameters in the structure shown in Fig. 6:

$$
\varphi=\frac{\pi d}{4 S} .
$$

In 3D structures such as metal foams, fibers can have any arbitrary orientation in space, [see Fig. 7(a)]. Following Jackson and James [26], the microstructure of 3D fibrous materials is modeled by a simple cubic (SC) arrangement. Figure 7(b) shows a SC structure used to model 3D media in this study and the transverse flow direction. The relationship between the solid volume fraction and other geometric parameters of SC arrangement considered in this study is [28]

$$
\varphi=\frac{3 \pi d^{2}}{4 S^{2}}-\sqrt{2} \frac{d^{3}}{S^{3}} .
$$

\section{EXPERIMENTAL APPROACH}

Experimental data for creeping flow through fibrous structures of our interests are not abundant in the open literature. As such, several samples of tube banks with 1D square

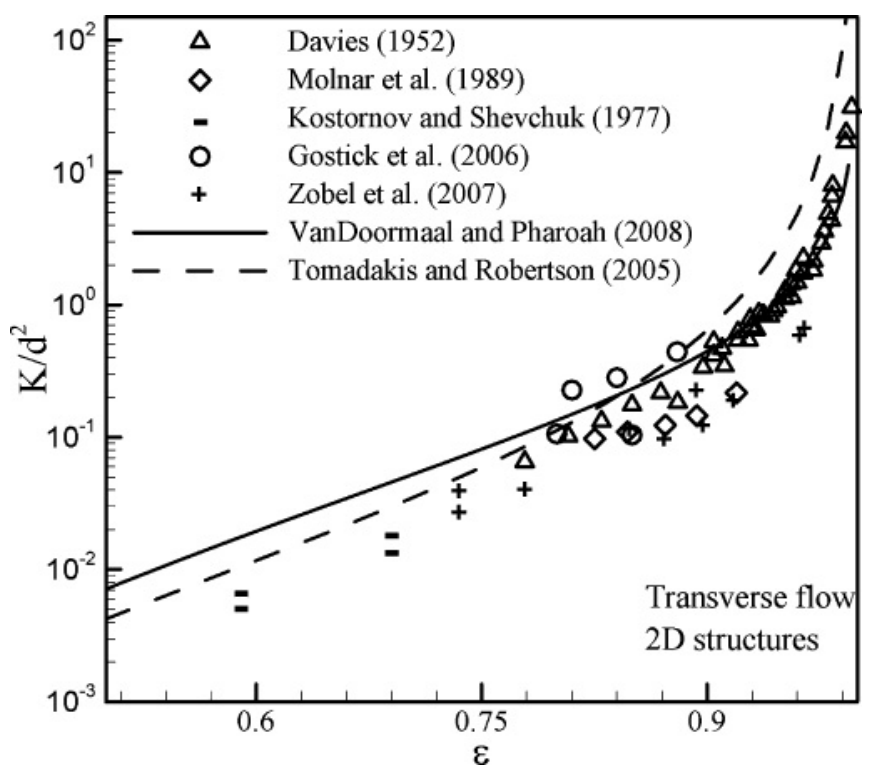

FIG. 3. Comparison of the existing models for 2D structures with experimental data reported by others. 


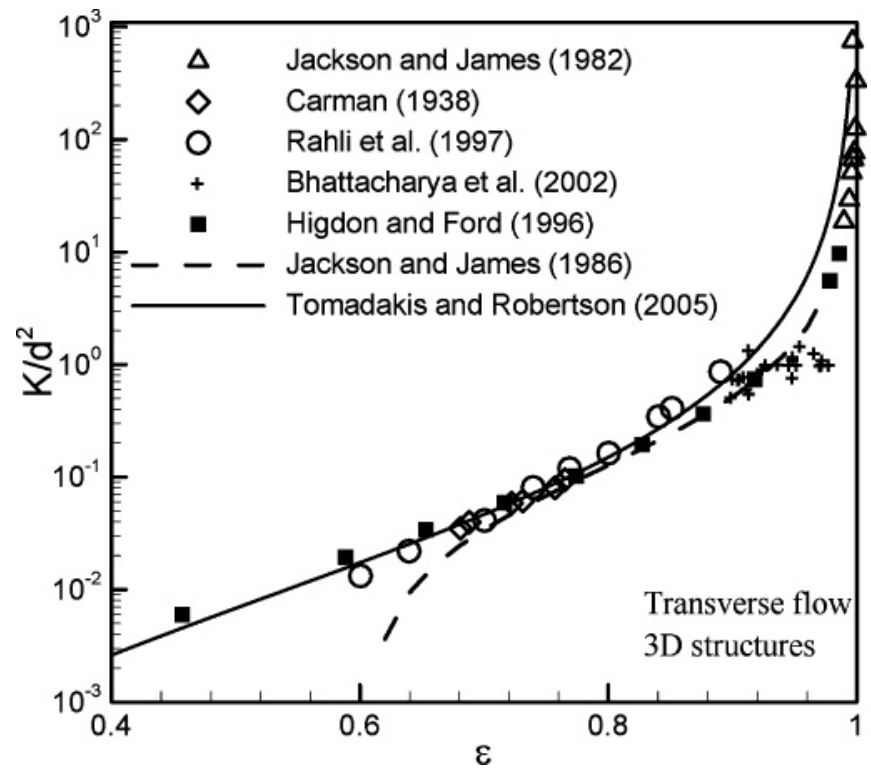

FIG. 4. Comparison of the existing models for 3D structures with experimental data for various materials.

and staggered fiber arrangements and metal foams with 3D microstructures shown in Fig. 8 are tested using glycerol. To fabricate the tube bank samples, polymethyl methacrylate (PMMA) sheets of 3-mm thickness were cut and drilled using a laser cutter with the accuracy of $0.05 \mathrm{~mm}$. Glass capillary tubes with diameters of $1.5 \mathrm{~mm}$ were inserted and fixed using an adhesive tape to form tube banks, as shown in Fig. 8. The lengths of the tube banks were selected such that a minimum of 15 rows of cylinders existed in the flow direction for each sample. Aluminum 6101 metal-foam samples were purchased from ERG Duocel (Oakland, CA) with a number of pores per inch (PPI) between 10 and 40. The porosity of the samples was also calculated independently by weighting the samples and measuring their volumes. The fiber diameters were estimated using scanning electron microscopy (SEM) images and also compared with the data reported by others [29] for similar materials. The properties of the samples are summarized in Table I.

A custom-made gravity-driven test bed, illustrated in Fig. 8, was built that included an elevated reservoir, an entry section, sample holder section, and an exit section with a ball valve. The reservoir cross section of $300 \times 300 \mathrm{~mm}^{2}$ was large enough to ensure that the variation of the pressure head was negligible during the experiment. The pressure drop across the samples was measured using a differential pressure transducer PX-154

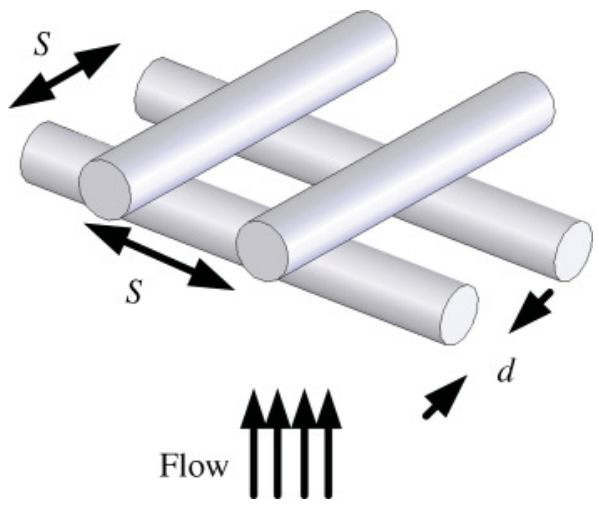

FIG. 6. (Color online) The 2D unit cell considered in the present study.

(BEC Controls) with $1 \%$ accuracy. To minimize the entrance and exit effects on the pressure-drop measurements, pressure taps were located few rows apart (at least three rows) from the first and the last tube rows in the tube bank samples and $1 \mathrm{~cm}$ apart from the sample edge for metal foams. Glycerol was used as the testing fluid and the bulk flow was calculated by weighting the collected test fluid over a period of time.

The Reynolds number was defined based on the fibers' diameter, i.e., $\operatorname{Re}=\rho U_{D} d / \mu$, and was kept below 0.001 to ensure creeping flow regime in the tested media. As such, the permeability of the samples was calculated using the Darcy equation (4) in the following section.

Assuming Darcy's law in a porous structure implies a linear relationship between the pressure drop and the fluid velocity in the media. This linear relationship can be observed in Fig. 9 for tube banks with square and staggered fiber arrangements and metal-foam samples.

\section{MODEL DEVELOPMENT}

Experimental observations have shown that a linear relationship exists between the volume-averaged superficial fluid velocity $U_{D}$ and the pressure drop; this is called Darcy's law [4]:

$$
-\frac{d P}{d x}=\frac{\mu}{K} U_{D}
$$

where $\mu$ is the fluid viscosity and $K$ is the permeability of the medium. Darcy's relationship is empirical, convenient, and widely accepted; this equation holds when flow is in creeping regime [4]. However, one should know the permeability beforehand to use the Darcy's equation. Permeability can be

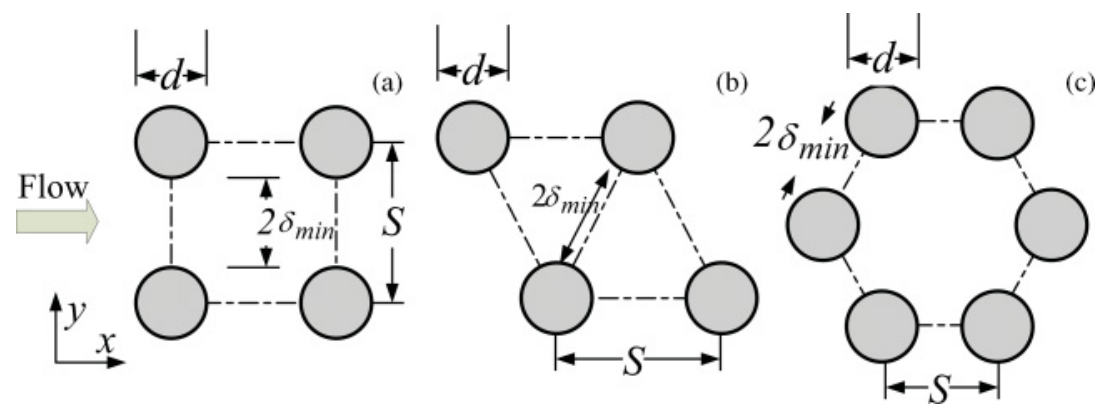

FIG. 5. (Color online) Considered unit cells for ordered 1D structures: (a) square, (b) staggered, and (c) hexagonal arrays of cylinders. 
(a)

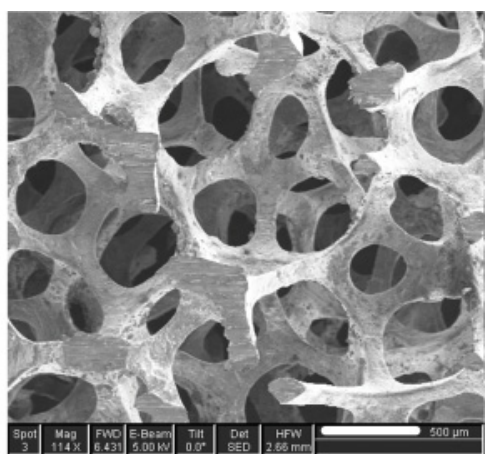

(b)

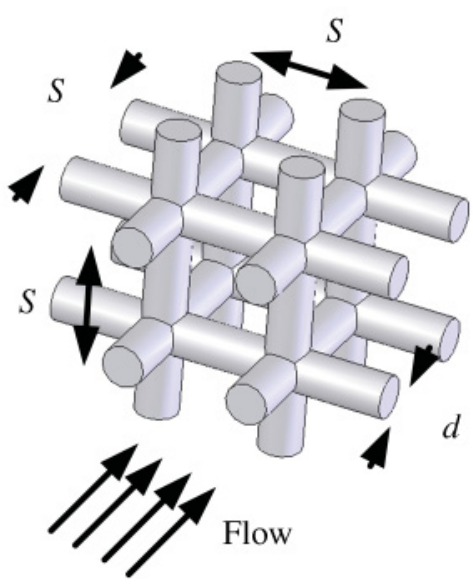

FIG. 7. (Color online) 3D structures: (a) metal foam, a real structure (scale bar is equal to $500 \mu \mathrm{m}$ ); (b) simple cubic arrangement, modeled unit cell used in this analysis.

calculated through the pore-scale analysis of flow in the solid matrix. In the creeping regime, the pore-scale velocity $\vec{V}$ is governed by Stokes equation

$$
\begin{gathered}
\vec{\nabla} \cdot \vec{V}=0, \\
\mu \nabla^{2} \vec{V}=-\vec{\nabla} P .
\end{gathered}
$$

A scale analysis is followed for determining the resulting pressure drop. In this approach, the scale or the range of variation of the parameters involved is substituted in governing equations, i.e., derivatives are approximated with differences [30]. Following Clague et al. [27] and Sobera and Kleijn [31], half of the minimum opening between two adjacent cylinders $\delta_{\min }$ is selected as the characteristic length scale over which rapid changes of velocity occur (see Fig. 5). Therefore, Eq. (6) scales as

$$
-\frac{\Delta P}{\ell} \sim \frac{\mu}{\delta_{\min }^{2}}|\vec{V}|,
$$

where $\ell$ is the characteristic length scale in the flow direction (see [31]) for more details. In case of nontouching cylinders with their axes perpendicular to the flow direction, the maximum velocity occurs in the section with minimum frontal area. Sobera and Klein [31] proposed to use the average velocity in the section with minimum frontal area as the characteristic velocity scale, i.e., $|\vec{V}| \sim U_{D} / \beta$; where $\beta$ is the ratio of the minimum to the total frontal areas in the unit cell. However, this assumption was only accurate for highly porous structures $\varepsilon>0.8$, and overpredicted the pressure drop in low porosities [31]. Carman [32] argued that a fluid particle should travel in a tortuous path of length $L_{e}$ to pass through a sample of size $L$. Therefore, it is expected that the resulting velocity scale for a constant pressure drop be inversely related to $L_{e} / L$; this ratio is called the tortuosity factor $\tau$. Thus, the scale of the pore-level velocity magnitude becomes

$$
|\vec{V}| \sim \frac{U_{D}}{\tau \beta} .
$$

Substituting from Eq. (8) for velocity scale and using $\delta_{\min }$ as the length scale, permeability can be calculated as

$$
K=C \beta \delta_{\min }^{2} \tau,
$$

where $C$ is a constant that should be determined through comparison with data. Therefore, one needs to know the ratio between the minimum to total frontal area $\beta$ and the tortuosity factor $\tau$ to be able to calculate the permeability.

\section{A. Tortuosity Factor}

The tortuosity factor is defined as the ratio of the average distance $L_{e}$ that a particle should travel in a media to cover a direct distance of $L$. Due to its importance in mass, thermal, and electrical diffusion in porous media, several theoretical and empirical relationships have been proposed for tortuosity calculation in the literature; good reviews can be found elsewhere [33,34]. Any relationship proposed for tortuosity should satisfy three conditions [33]: $\tau>1 ; \lim _{\varepsilon \rightarrow 1} \tau=1$; $\lim _{\varepsilon \rightarrow 0} \tau \rightarrow \infty$. One of the most popular empirical models for determination of tortuosity, which satisfies all these conditions, is Archie's law [35]

$$
\tau=\left(\frac{1}{\varepsilon}\right)^{\alpha}=\left(\frac{1}{1-\phi}\right)^{\alpha},
$$

where $\alpha$ is a constant and $\varepsilon$ is the porosity. $\alpha$ is a tuning parameter that is found through comparison of Archie's

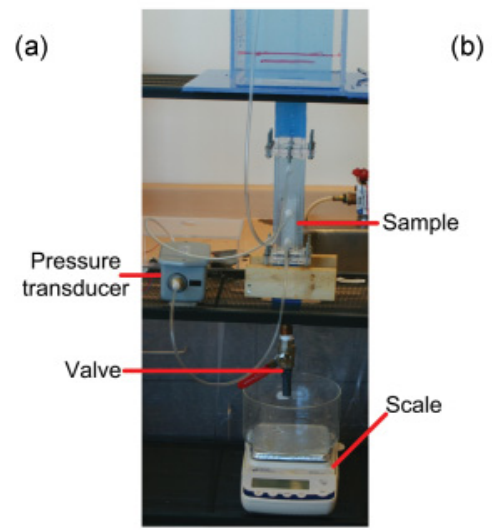

(b)

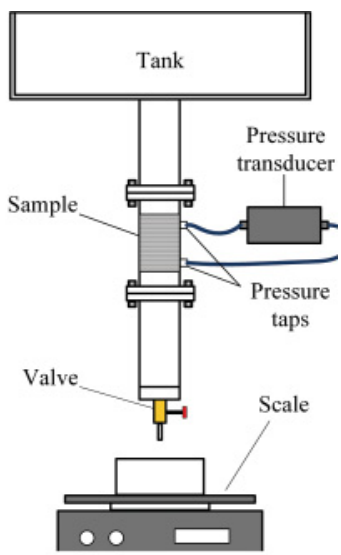

(c)

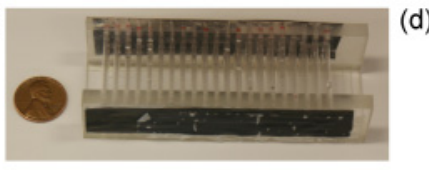

(d)

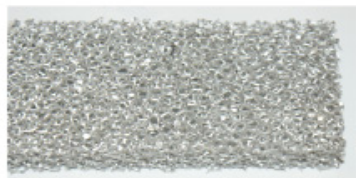

FIG. 8. (Color online) The gravity-driven test bed and the tested samples: (a) actual test setup, (b) schematic of the apparatus; (c) a sample of ordered tube banks; and (d) a sample of aluminum foam. 
TABLE I. The properties of the tested samples.

\begin{tabular}{llcccc}
\hline \hline Sample type & $\varepsilon$ & $d(\mathrm{~mm})$ & $L(\mathrm{~mm})$ & Orientation & Measured permeability $K\left(\mathrm{~m}^{2}\right)$ \\
\hline Tube bank (square) & 0.8 & 1.5 & 69 & $1 \mathrm{D}$ & $1.38 \times 10^{-7}$ \\
Tube bank (square) & 0.85 & 1.5 & 80 & $1 \mathrm{D}$ & $3.74 \times 10^{-7}$ \\
Tube bank (square) & 0.9 & 1.5 & 68 & $1 \mathrm{D}$ & $5.44 \times 10^{-7}$ \\
Tube bank (staggered) & 0.7 & 1.5 & 74 & $1 \mathrm{D}$ & $1.00 \times 10^{-7}$ \\
Tube bank (staggered) & 0.9 & 1.5 & 72 & $1 \mathrm{D}$ & $7.75 \times 10^{-7}$ \\
Metal foam (PPI = 10) & 0.93 & 0.4 & 137 & $3 \mathrm{D}$ & $2.53 \times 10^{-7}$ \\
Metal foam (PPI = 20) & 0.93 & 0.3 & 135 & $3 \mathrm{D}$ & $1.45 \times 10^{-7}$ \\
Metal foam (PPI =40) & 0.94 & 0.2 & 120 & $3 \mathrm{D}$ & $0.81 \times 10^{-7}$ \\
\hline \hline
\end{tabular}

empirical correlation, i.e., Eq. (10), with experimental data. Boudreau [33], through comparison with data, showed that $\alpha=0.5$ provides a reasonable estimate for tortuosity in packed beds. The axes of fibers in 1D and 2D microstructures are perpendicular to the transverse flow direction, which is similar to the flow through packed beds of spherical particles. As a result, $\alpha=0.5$ provides a good estimate for the tortuosity of 1D and 2D structures as well. However, in 3D structures, some of the fibers (roughly $1 / 3$, consider an equally spaced equally sized simple cubic unit cell) are parallel to the flow direction [36] and do not affect the tortuosity of the medium. The study of Tomadakis and Sotirchos [12] also showed that $3 \mathrm{D}$ fibrous structures are less tortuous in comparison with 1D and 2D matrices. Consequently, an $\alpha$ smaller than 0.5 should be used for 3D microstructures. The deviation of Archie's law with $\alpha=0.3$ from the tortuosity values predicted by the model of Tomadakis and Sotirchos [24] is less than 20\%. In this paper, our model for permeability of 3D structures $(\alpha=$ 0.3 ) and the present experimental results and the data collected from various sources are in good agreement.

\section{RESULTS AND DISCUSSIONS}

Equation (9) relates the permeability of fibrous media to the minimum opening between adjacent fibers $\delta_{\min }$, the ratio between minimum to total frontal area $\beta$, and tortuosity factor $\tau$, which can be calculated from Eq. (10). In the following sections, using geometrical properties of the considered microstructures, compact models will be developed that relate the permeability to the solid volume fraction.

\section{A. Unidirectionally Aligned Arrangements}

For the three different ordered 1D unit cells shown in Fig. 5, it can be seen that $\beta=(S-d) / S$ and $\delta_{\min }=(S-d) / 2$. Therefore, Eq. (9) can be rewritten as

$$
K=C \frac{(S-d)^{3}}{S \sqrt{1-\phi}}
$$

TABLE II. The average relative difference between the permeability values predicted by different models with the numerical and experimental data for 1D square, 1D staggered, and 3D simple cubic structures over the entire range of porosity.

1D square arrays

(compared to experimental data)

\begin{tabular}{llll}
\hline Author(s) & $\begin{array}{l}\text { Relative } \\
\text { difference (\%) }\end{array}$ & Author(s) & $\begin{array}{l}\text { Relative } \\
\text { difference (\%) }\end{array}$ \\
\hline Tamayol and Bahrami [21] & 24.9 & Happel [15] & 47.5 \\
Gebart [23] & 26.8 & Drummond and Tahir [19] & 245.2 \\
Van der Westhuizen [20] & 45.8 & Present scale analysis & 15.8 \\
Sahraoui and Kaviany [22] & 25.6 & & \\
\hline
\end{tabular}

1D staggered arrays

(compared to present experimental data and numerical results of Higdon and Ford [28])

$\begin{array}{llcl}\text { Gebart [23] } & 11.6 & \text { Present scale analysis } & 26.6 \\ \text { Happel [15] } & 38.8 & \end{array}$

3D simple cubic structure

(compared to numerical results of Higdon and Ford [28])

Tomadakis and Robertson [12]

Jackson and James [26]
Present scale analysis 
Combining Eqs. (1) and (11), the dimensionless permeability of the ordered structures becomes

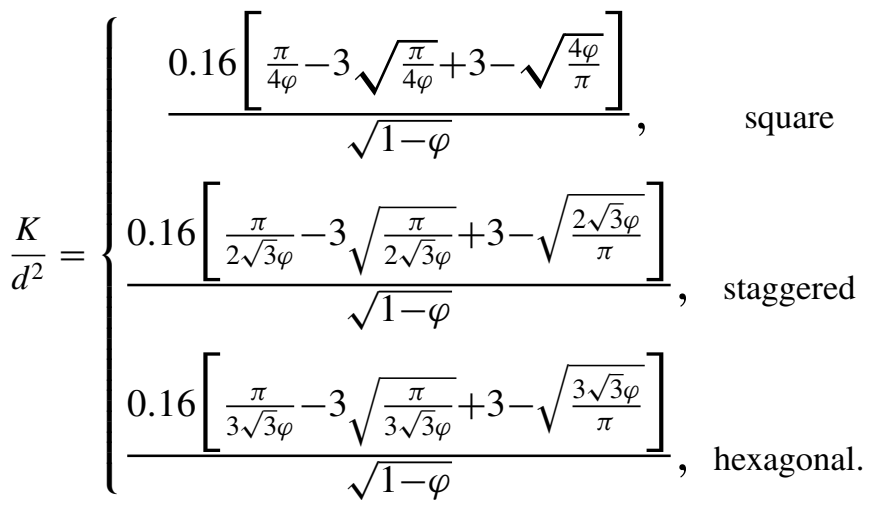

The constant values in Eq. (12), 0.16, are evaluated through comparison of the proposed model with the experimental and numerical data found in the literature. In Fig. 10, Eq. (12) is compared with the present experimental results and the data collected by others [37-43]. As one can see, the model is in agreement with experimental data over the entire range of porosity. These experiments were conducted using different fluids including air, water, oil, and glycerol with a variety of porous materials such as metallic rods, acrylic cylinders, and carbon fibers.

In Fig. 11, the predicted results of Eq. (12) for staggered arrangement of fibers are compared with the present experimental data and numerical results of Higdon and Ford [28]. It can be seen that the proposed model can accurately predict the numerical results in the entire range of porosity. The average relative differences between the numerical and experimental data with the values predicted by various models for $1 \mathrm{D}$ structures are reported in Table II.

\section{B. Two-Directional Structures}

The ratio of the minimum frontal to the total unit-cell areas for the 2D structure, shown in Fig. 6, is not exactly known. Therefore, using the Forchheimer law, which estimates the average pore-scale velocity as $U_{D} / \varepsilon$ [4], the magnitude of the pore-level velocity scale is estimated as

$$
|\vec{V}| \approx \frac{U_{D}}{\tau \varepsilon} .
$$

The length scale, where the rapid changes of the velocity occur, is assumed as $\delta_{\min }=(S-d) / 2$. Therefore, the permeability reads as

$$
K=C(S-d)^{2} \varepsilon \tau
$$

Substituting for geometrical parameters from Eq. (2) and the tortuosity from Archie's law, the dimensionless permeability becomes

$$
\frac{K}{d^{2}}=0.008 \sqrt{(1-\varphi)}\left[\left(\frac{\pi}{4 \varphi}\right)^{2}-2 \frac{\pi}{4 \varphi}+1\right] .
$$

(a)

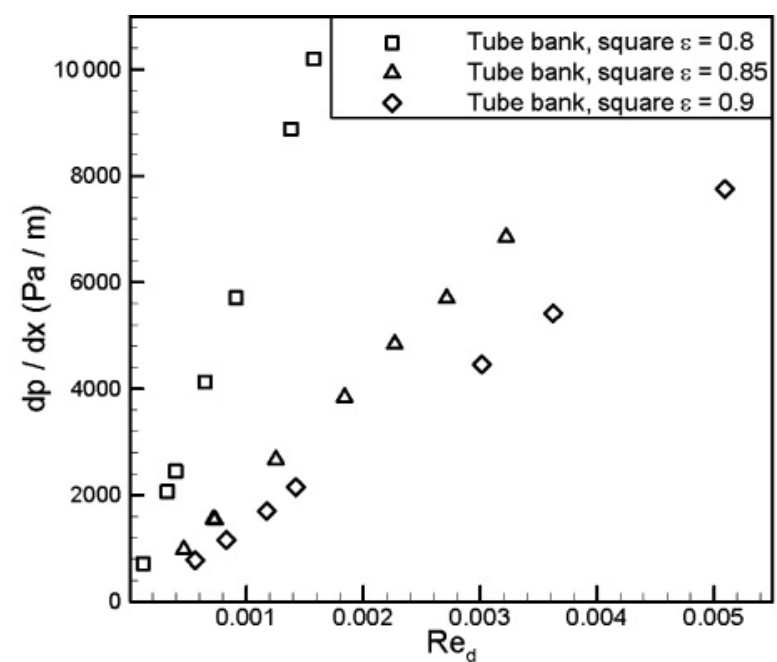

(b)

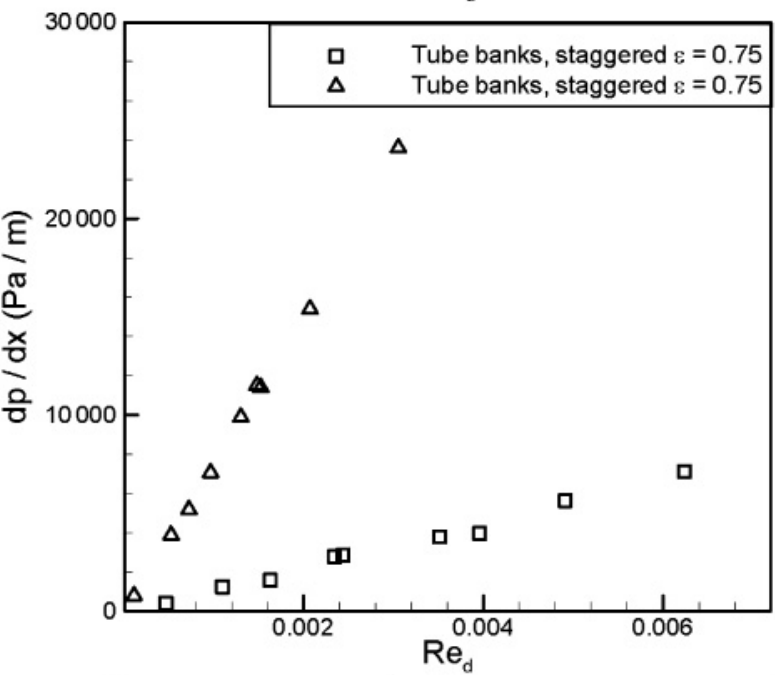

(c)

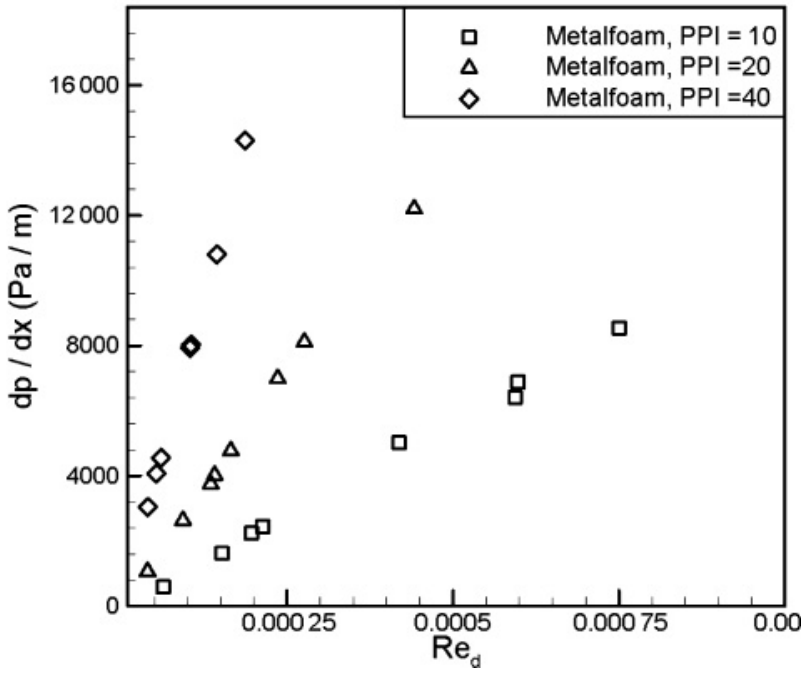

FIG. 9. Measured pressure drop for samples of tube bank with square fiber arrangement.

The constant value in Eq. (15), i.e., 0.008, is found through comparison with experimental data collected from different sources (see Fig. 12). It can be seen that Eq. (15) captures the trends of the experimental data collected from different sources over a wide range of porosity. The experiments were conducted 


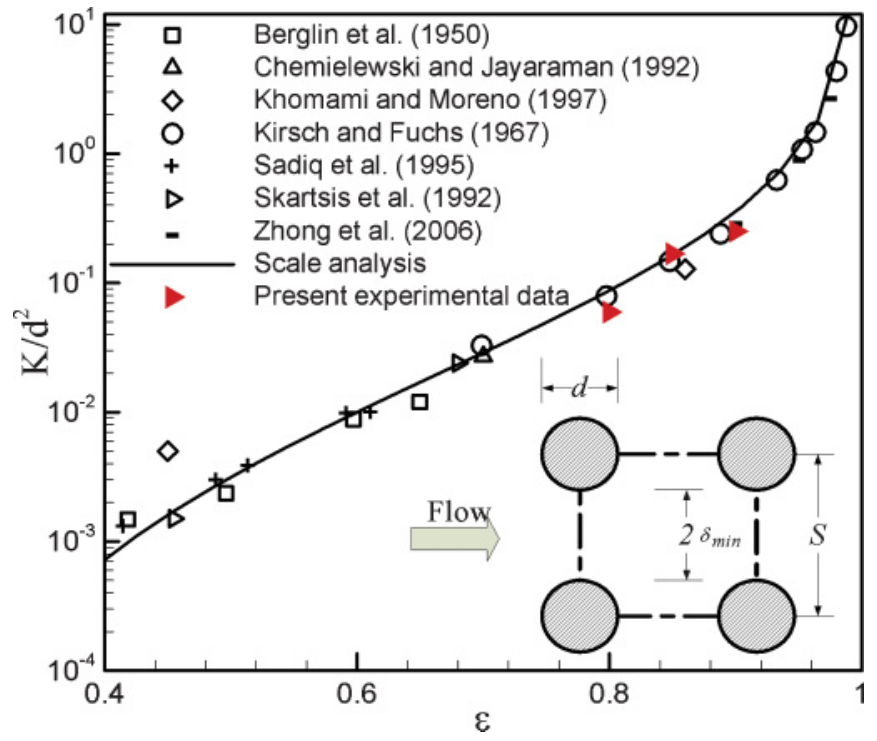

FIG. 10. (Color online) Comparison of the proposed model for square arrangements with experimental data measured in this paper or reported by others.

on glass rods, glass wool, cotton wool, kapok with application in filtration [44], alloy fibers [45], fiber reinforcing mats with application in molding and composite fabrication [46,47], and gas diffusion layers [10]. Kostornov and Shevchuk [45] performed experiments with several fluids and they observed that the permeability was dependent on the working fluid, i.e., water resulted in higher permeability than alcohol. Models of Tomadakis and Robertson [12] and Van Doormaal and Pharoah [25] are also compared with Eq. (15) in Fig. 12. For highly porous materials $(\varepsilon>0.8)$, the correlation proposed by Van Doormaal and Pharoah [25] also accurately predicts the experimental data, while the model of Tomadakis and

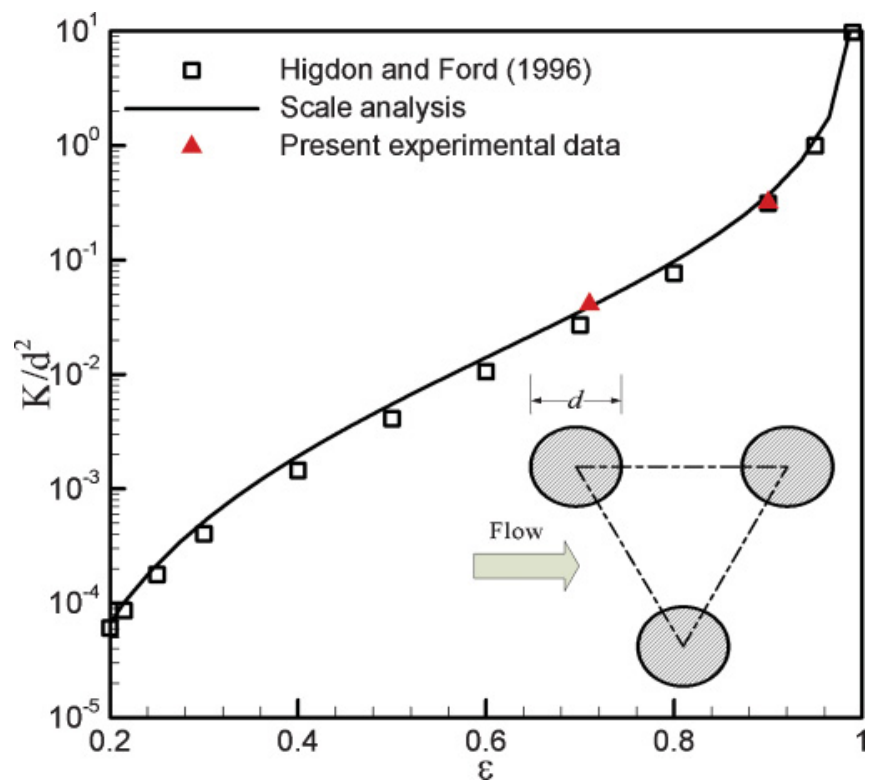

FIG. 11. (Color online) Comparison of the proposed model and current experimental data with numerical results of Higdon and Ford [28] for staggered arrangements.

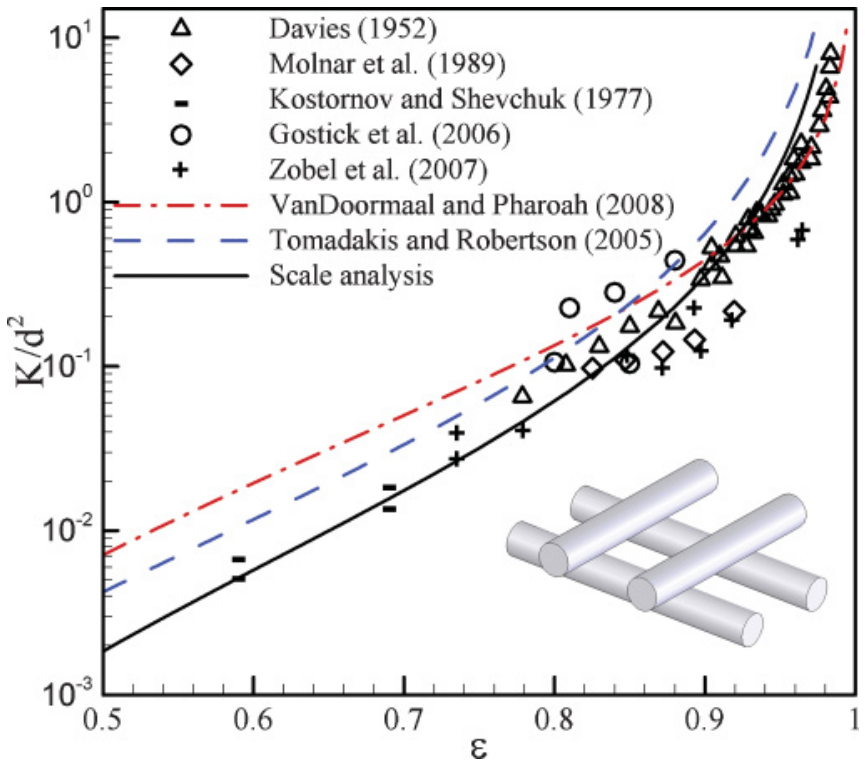

FIG. 12. (Color online) Comparison of this model and models of Van Doormaal and Pharoah [25] and Tomadakis and Robertson [12] with experimental data for $2 \mathrm{D}$ structures.

Robertson [12] captures the trends of experimental data in lower porosities.

\section{Three-Directional Structures}

For simple cubic arrangement that is considered in this study as a simple representation of 3D fibrous materials, the ratio of the minimum frontal to the unit-cell areas is $\beta=(S-$ $d)^{2} / S^{2}$ and $\delta_{\min }=(S-d) / 2$. Therefore, the permeability of

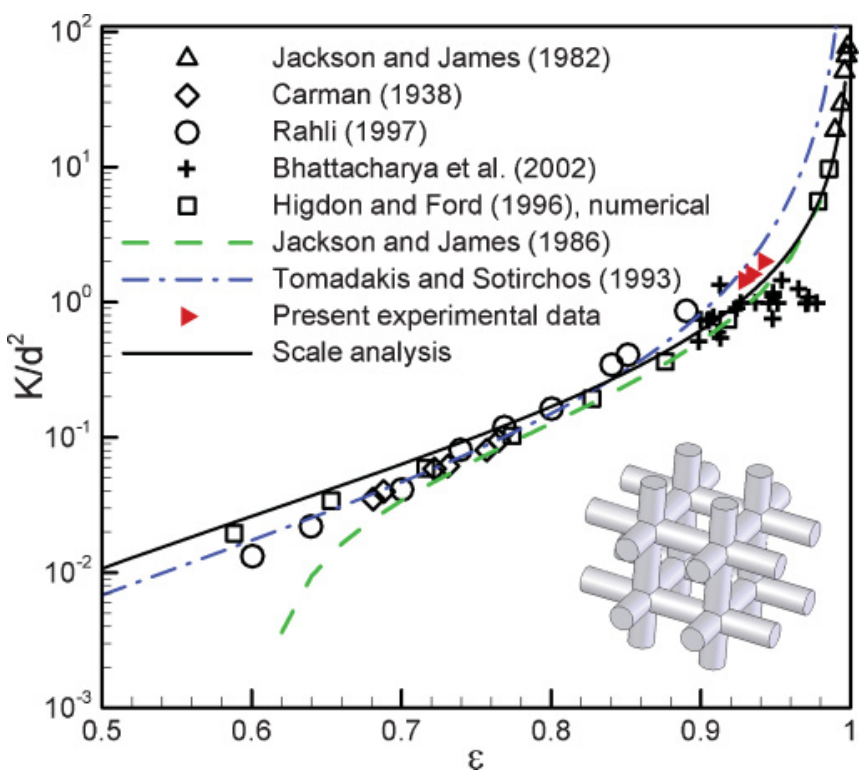

FIG. 13. (Color online) Comparison of the proposed model for 3D structures, models of Jackson and James [26] and Tomadakis and Robertson [12], present experimental results, and data reported by others. 


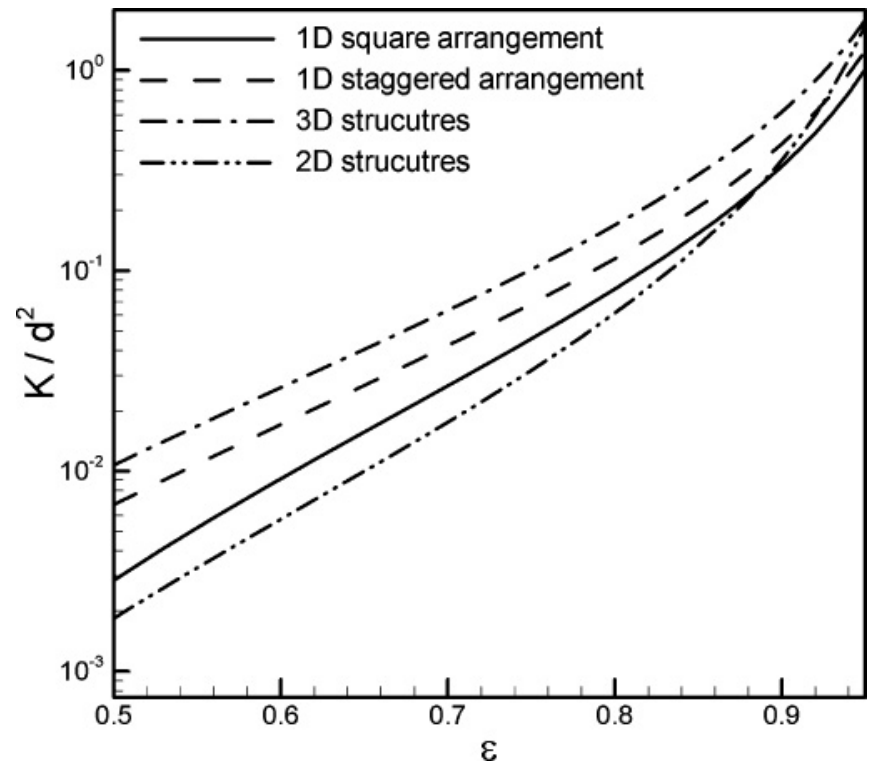

FIG. 14. Effect of fiber orientation on the permeability of fibrous structures.

3D structures becomes

$$
\frac{K}{d^{2}}=0.08 \frac{(S-d)^{4}}{S^{2} d^{2} \varepsilon^{0.3}},
$$

where the ratio of $S$ to $d$ is calculated from Eq. (3). The constant in Eq. (16) is found to be 0.08 through comparison of this equation with the numerical data reported by Higdon and Ford [28] for SC arrangements over a wide range of porosity. Figure 13 includes the present model, models of Tomadakis and Robertson [12] and Jackson and James [26], current experimental measurements, and experimental data collected from different sources. The plotted data are based on the permeability values reported for polymer chain in solutions [48], glass wool randomly packed, stainless-steel crimps [32], metallic fibers [49], and aluminum metal foams [50]. It can be seen that the present model is in agreement with the experimental data and numerical results collected from a number of studies over the entire range of porosity. The average relative differences between the numerical data reported by Higdon and Ford [28] and Eq. (15), the model of Tomadakis and Robertson [12], and the model of Jackson and James [26] are listed in Table II.

\section{EFFECT OF FIBER ORIENTATION ON THE PERMEABILITY}

To investigate the effect of the fibers' arrangement on the permeability of the fibrous structures, the proposed relationships for 1D, 2D, and 3D arrays are plotted in Fig. 14. It can be seen that the square arrangements and $2 \mathrm{D}$ structures have similar permeabilities and 3D structures are the most permeable microstructure; this is in agreement with the results reported by Tomadakis and Robertson [12]. Moreover, the effect of microstructure is more significant in low porosities, where $\varepsilon<0.7$ and the deviations become less pronounced in higher porosities; this is in line with our previous observations for parallel flow through 1D fibers [51].

\section{CONCLUSIONS}

A scale analysis technique was employed for analyzing pressure drop and permeability of fibrous media. The fibrous materials were represented by unit cells that were assumed to be repeated throughout the medium and the present approach was applied to a variety of fibrous matrices including square, staggered, and hexagonal unidirectionally aligned fiber arrangements, as well as simple two- directional mats and simple cubic structures. Moreover, compact relationships have been reported for the considered geometries. In addition, pressure drop in samples of tube banks of different arrangements and aluminum foams with various PPIs were measured in the creeping flow regime. The developed compact relationships were successfully verified through comparison with a large number of experimental and numerical data collected from various sources, over a wide range of porosity. Our results indicated that the microstructural effects were more significant for low porosities. Moreover, 3D structures had the highest permeability in comparison with 1D and 2D structures.

Since the present model relates permeability to the tortuosity, fiber size, and distribution, one can conclude that it can potentially be extended to include randomness effects in real fibrous porous structures.

\section{ACKNOWLEDGMENTS}

The authors gratefully acknowledge the financial support of the British Columbia Innovation Council (BCIC) and the Natural Sciences and Engineering Research Council of Canada (NSERC).
[1] G. A. Truskey, F. Yuan, and D. E. Katz, Transport Phenomena in Biological Systems (Prentice Hall, New Jersey, 2004).

[2] L. Spielman, S. L. Goren, Environ. Sci. Technol. 2, 279 (1968).

[3] F. A. L. Dullien, Porous Media: Fluid Transport and Pore Structure (Academic, New York, 1992).

[4] M. Kaviany, Principles of Heat Transfer in Porous Media (Springer, New York, 1992).

[5] M. A. Choi, M. H. Lee, J. Chang, and S. J. Lee, J. Non-Newtonian Fluid Mech. 79, 585 (1999).

[6] B. T. Astrom, R. B. Pipes, and S. G. Advani, J. Compos. Mater. 26, 1351 (1992).
[7] L. Tadrist, M. Miscevic, O. Rahli, and F. Topin, Exp. Therm. Fluid Sci. 28, 193 (2004).

[8] S. Mahjoob and K. Vafai, Int. J. Heat Mass Transfer 51, 3701 (2008).

[9] C. Antoine, P. Nygard, Ø. W. Gregersen, R. Holmstad, T. Weitkamp, and C. Rau, Nucl. Instrum. Methods Phys. Res. A 490, 392(2002).

[10] J. P. Feser, A. K. Prasad, and S. G. Advani, J. Power Sources 162, 1226 (2006).

[11] J. T. Gostick, M. W. Fowler, M. D. Pritzker, M. A. Ioannidis, and L. M. Behra, J. Power Sources 162, 228 (2006). 
[12] M. M. Tomadakis and T. Robertson, J. Compos. Mater. 39, 163 (2005).

[13] T. M. Quinn, P. Dierickx, and A. J. Grodzinsky, J. Biomech. 38 1483 (2001).

[14] S. Federico and W. Herzog, Int. J. Solids Struct. 45, 2160 (2008).

[15] J. Happel, AIChE J. 5, 174 (1959).

[16] S. Kuwabara, J. Phys. Soc. Jpn. 14, 527 (1959).

[17] H. Hasimoto, J. Fluid Mech. 5, 317 (1959).

[18] A. S. Sangani and A. Acrivos, Int. J. Multiphase Flow 8, 193 (1982).

[19] J. E. Drummond and M. I. Tahir, Int. J. Multiphase Flow 10, 515 (1984).

[20] J. Vander Westhuizen and J. P. Du Plessis, Composites, Part A 27, 263 (1996).

[21] A. Tamayol and M. Bahrami, Int. J. Heat Mass Transf. 52, 2407 (2009).

[22] M. Sahraoui and M. Kaviany, Int. J. Heat Mass Transf. 37, 1029 (1994).

[23] B. R. Gebart, J. Compos. Mater. 26, 1100 (1992).

[24] M. M. Tomadakis and S. V. Sotirchos, J. Chem. Phys. 98, 616 (1993).

[25] M. A. Van Doormaal and J. G. Pharoah, Int. J. Numer. Methods Fluids 59, 75 (2009).

[26] G. W. Jackson and D. F. James, Can. J. Chem. Eng. 64, 364 (1986).

[27] D. S. Clague, B. D. Kandhai, R. Zhang, and P. M. A. Sloot, Phys. Rev. E 61, 616 (2000).

[28] J. J. L. Higdon and G. D. Ford, J. Fluid Mech. 308, 341 (1996).

[29] V. V. Calmidi and R. L. Mahajan, J. Heat Transfer 122, 557 (2000).

[30] F. M. White, Viscous Fluid Flow (McGraw-Hill, New York, 1984).

[31] M. P. Sobera and C. R. Kleijn, Phys. Rev. E 74, 036301 (2006).
[32] P. C. Carman, Chem. Eng. Res. Des. 15a, 150 (1937).

[33] B. P. Boucreau, Geometrica Cosmochim. Acta 60, 3139 (1996).

[34] L. Shen and Z. Chen, Chem. Eng. Sci. 62, 3748 (2007).

[35] G. Archie, Trans. AIME 146, 54 (1942).

[36] A. Tamayol and M. Bahrami, J. Power Sources 196, 3559 (2011).

[37] O. P. Bergelin, G. A. Brown, H. L. Hull, and F. W. Sullivan, Trans. ASME 72, 881 (1950).

[38] O. P. Bergelin, G. A. Brown, H. L. Hull, and F. W. Sullivan, J. Heat Transfer 72, 881 (1950).

[39] A. A. Kirsch and N. A. Fuchs, The Annals of Occupational Hygiene 10, 23 (1967).

[40] B. Khomami and L. D. Moreno, Rheol. Acta 36, 367 (1997).

[41] L. Skartsis, B. Khomami, and J. L. Kardos, Polym. Eng. Sci. 32, 231 (1992).

[42] W. H. Zhong, I. G. Currie, and D. F. James, Exp. Fluids 40, 119 (2006).

[43] C. Chmielewski and K. Jayaramana, J. Rheol. (Melville, NY, US) 36, 1105 (1992).

[44] C. N. Davies, Institute of Mechanical Engineers, London B1, 185 (1952).

[45] A. G. Kostornov and M. S. Shevchuk, Powder Metall. Met. Ceram. 16, 694 (1977).

[46] J. A. Molnar, L. Trevino, and L. J. Lee, Polym. Compos. 10, 414 (1989).

[47] S. Zobel, B. Maze, H. Vahedi Tafreshi, Q. Wang, and B. Pourdeyhimi, Chem. Eng. Sci. 62, 6285 (2007).

[48] G. W. Jackson and D. F. James, Biorheology 19, 317 (1982).

[49] O. Rahli, L. Tadrist, M. Miscevic, and R. Santini, J. Fluids Eng. 119, 188 (1997).

[50] A. Bhattacharya, V. V. Calmidi, and R. L. Mahajan, Int. J. Heat Mass Transfer 45, 1017 (2002).

[51] A. Tamayol and M. Bahrami, J. Fluids Eng. (ASME) 132, 114502 (2010). 